\title{
Earlier diagnosis of breast cancer: focusing on symptomatic women
}

Georgios Lyratzopoulos and Gary Abel

Amidst the rigorous breast cancer screening debate, as commented on by Autier et al. (Viewpoint: Breast cancer screening: the questions answered. Nat. Rev. Clin. Oncol. 9, 599-605; 2012) and others, ${ }^{1,2}$ it is easy to forget that two thirds of all women with breast cancer are diagnosed after presenting to their clinician with symptoms and not through screening. ${ }^{3}$ What are the opportunities for earlier diagnosis of breast cancer in these women?

Evidence indicates that older women (that is, those aged 70 years or older) and those in lower socioeconomic groups are at higher risk of being diagnosed with breast cancer at an advanced disease stage than their younger and wealthier counterparts. ${ }^{4,5}$ These inequalities, in part, reflect the lower uptake of screening among women of lower socioeconomic status, ${ }^{5,6}$ and the fact that older women are not routinely invited for screening. ${ }^{7}$ However, substantial inequalities remain even after adjusting for the confounding effect of screening: if it were possible to eliminate older age and deprivation inequalities nearly 1,000 women in England every year who are currently diagnosed with advanced-stage breast cancer would be diagnosed at an earlier stage. ${ }^{8}$ Revisiting this analysis suggests that about $90 \%$ of these gains would occur in symptomatically detected women. This figure represents more than two women being diagnosed with potentially avoidable advanced-stage breast cancer in England every day, or about one in seven women who are currently diagnosed in the advanced stages of disease. These are hard-to-ignore statistics. Preventing advanced-stage diagnosis among symptomatically detected women is a public health priority.

The association between delay and advanced stage at diagnosis is well documented for breast cancer. ${ }^{9}$ But, do delays occur after presentation of a symptomatic woman to a primary care physician, or prepresentation? Primary care delays for women subsequently diagnosed with breast cancer are trivial: both patient-reported and national audit data indicate that referrals occur promptly, indeed most women are referred as soon as they present. ${ }^{10-12}$ Furthermore, there is no evidence that post-presentation delays are influenced by deprivation status, and primary care physicians suspect cancer more promptly in older, not younger, patients. ${ }^{10}$ Therefore, it is highly unlikely that age and deprivation inequalities in the stage at diagnosis of breast cancer reflect inequalities in delays after presentation.

These data sharply focus attention on potential inequalities in prepresentation delay, because of patient factors such as differential awareness, beliefs, and attitudes to cancer symptoms. ${ }^{13,14}$ Measuring 'patient delay' (from symptom onset to help-seeking) accurately is difficult, but research has established socioeconomic and age gradients in the awareness of common cancer symptoms. ${ }^{15}$ Both cognitive and psychological factors might be implicated in differential prepresentation delay among different patient groups. ${ }^{16}$ Among women aged 70 years or older, inequalities in stage at diagnosis might represent a cohort effect or a tendency for older women (including those women who have undergone breast cancer screening) to downplay breast cancer risk in later life. Either way, stratified (that is, targeted and tailored) interventions for older and deprived women are justified. Regarding age inequalities, such a stratified approach has been shown to improve interim outcomes, such as improved awareness. ${ }^{17}$ Furthermore, it is encouraging that the current 'be clear on cancer' initiative in the UK particularly focuses on breast cancer in older women. ${ }^{18}$ However, if substantial inroads are to be achieved in reducing inequalities in stage at diagnosis, interventions other than those based on written material, and focusing on women of different socioeconomic strata need to be developed. We should not let the rigorous debate on breast cancer screening divert attention from real opportunities to diagnose breast cancer earlier and reduce inequalities for symptomatically detected women.

Cambridge Centre for Health Services Research, Institute of Public Health, University of Cambridge, Forvie Site, Robinson Way, Cambridge CB2 OSR, UK (G. Lyratzopoulos, G. Abel).

Correspondence to: G. Lyratzopoulos gl290@medschl.cam.ac.uk

Acknowledgements

G. Lyratzopoulos is supported by a Post-Doctoral Fellowship award by the National Institute for Health Research (NIHR PDF-2011-04-047). The views expressed are those of the authors and not necessarily those of the NHS, the National Institute for Health Research or the Department of Health. The funder had no role in this paper's conception, writing and the decision to submit it for publication.

\section{Competing interests}

G. Lyratzopoulos is a member of the following professional groups: NICE Guidelines Development Group for 'Referral for Suspected Cancer' and NCRI Clinical Studies Group for Primary Care. G. Abel declares no competing interests.

1. Autier, P., Esserman, L. J., Flowers C. I. \& Houssami, N. Breast cancer screening: the questions answered. Nat. Rev. Clin. Oncol. 9, 599-605 (2012).

2. [No authors listed]. The breast cancer screening debate: closing a chapter? Lancet 380, 1714 (2012).

3. Cancer Research UK. Breast cancer Key Facts. Cancer Research UK Publications [online], http://publications.cancerresearchuk.org/ cancerstats/statsbreast/kfbreast.html (2013).

4. Adams, J., White, M. \& Forman, D. Are there socioeconomic gradients in stage and grade of breast cancer at diagnosis? Cross sectional analysis of UK cancer registry data. BMJ 329, 142 (2004).

5. Cuthbertson, S. A., Goyder, E. C. \& Poole, J. Inequalities in breast cancer stage at diagnosis in the Trent region, and implications for the NHS Breast Screening Programme. J. Public Health (Oxf.) 31, 398-405 (2009).

6. Harper, S. et al. Trends in area-socioeconomic and race-ethnic disparities in breast cancer incidence, stage at diagnosis, screening, mortality, and survival among women ages 50 years and over (1987-2005). Cancer Epidemiol. Biomarkers Prev. 18, 121-131 (2009). 


\section{CORRESPONDENCE}

7. Public Health England. NHS Breast Screening Programme [online], http://www. cancerscreening.nhs.uk/breastscreen (2013).

8. Lyratzopoulos, G. et al. Socio-demographic inequalities in stage of cancer diagnosis: evidence from patients with female breast, lung, colon, rectal, prostate, renal, bladder, melanoma, ovarian and endometrial cancer. Ann. Oncol. 24, 843-850 (2013).

9. Richards, M. A., Westcombe, A. M., Love, S. B., Littlejohns, P. \& Ramirez, A. J. Influence of delay on survival in patients with breast cancer: a systematic review. Lancet 353, 1119-1126 (1999).

10. Baughan, P., O’Neill, B. \& Fletcher, E. Auditing the diagnosis of cancer in primary care: the experience in Scotland. Br. J. Cancer 101 (Suppl. 2), S87-S91 (2009).

11. Lyratzopoulos, G., Neal, R. D., Barbiere, J. M., Rubin, G. P. \& Abel, G. A. Variation in number of general practitioner consultations before hospital referral for cancer: findings from the 2010 National Cancer Patient Experience Survey in England. Lancet Oncol. 13, 353-365 (2012).

12. Lyratzopoulos, G., Abel, G. A., McPhail, S. Neal, R. D. \& Rubin, G. P. Measures of promptness of cancer diagnosis in primary care: secondary analysis of national audit data on patients with 18 common and rarer cancers. Br. J. Cancer 108, 686-690 (2013).

13. Simon, A. E., Waller, J., Robb, K. \& Wardle, J. Patient delay in presentation of possible cancer symptoms: the contribution of knowledge and attitudes in a population sample from the United Kingdom. Cancer Epidemiol. Biomarkers Prev. 19, 2272-2277 (2010).

14. Niederdeppe, J. \& Levy, A. G. Fatalistic beliefs about cancer prevention and three prevention behaviors. Cancer Epidemiol. Biomarkers Prev. 16, 998-1003 (2007).

15. Robb, K. et al. Public awareness of cancer in Britain: a population-based survey of adults. Br. J. Cancer 101 (Suppl. 2), S18-S23 (2009).

16. Walter, F., Webster, A., Scott, S. \& Emery, J. The Andersen Model of Total Patient Delay: a systematic review of its application in cancer diagnosis. J. Health Serv. Res. Policy 17, 110-118 (2012).

17. Forbes, L. J. et al. A promoting early presentation intervention increases breast cancer awareness in older women after 2 years: a randomised controlled trial. $\mathrm{Br}$. J. Cancer 105, 18-21 (2011).

18. Department of Health. Be clear on cancer. NHS Choices [online], http://www.nhs.uk/ be-clear-on-cancer/Pages/beclearoncancer. aspx (2013). 